\title{
Urine Culture Collected from Gel-Based Diapers: Developing a Novel Experimental Laboratory Method
}

Pesach Shvartzman, MD, and Yussuf Nasri, MD

Background: Urinary tract infection diagnosis is based on urine culture, taken from a midstream collection. Obtaining samples in this manner is difficult in elderly patients suffering from incontinence and in infants.

Objectives: (1) Develop a method for urine collection using gel-based diapers and (2) compare culture results from gel-based diapers with those of the same urine, examined by accepted methods.

Methods: Urine was collected and cultured by standard bacteriologic techniques at the microbiology laboratory of Soroka Medical Center, Beer-Sheva, Israel. Gel-based diapers were manually dampened with the same urine samples, and samples from diapers were cultured 0 to 3 hours after dampening.

Results: Comparison showed good correlation between direct urine cultures and cultures obtained from gel based diapers $(R=1.000)$. Total sensitivity was $100 \%$ and specificity $97 \%$.

Conclusions: (1) Urine can be cultured from gel-based diapers and (2) a larger sample of in vivo trials will be needed to make this technique clinically applicable in an outpatient setting. ( $\mathrm{J}$ Am Board Fam Pract 2004;17:91-5.)

Urinary tract infection is a common clinical problem in the elderly population, infants, and young children. The diagnosis of urinary tract infection is based on a urine sample taken for culture, traditionally, from a midstream collection. Obtaining samples in this manner is difficult or impossible in elderly patients suffering from incontinence and from infants. Among the former, a urine sample is obtained by catheterization. This procedure is associated with pain and discomfort and in some cases with induced urinary tract infections. ${ }^{1-2}$ In infants and young children, urine is obtained by sterile adhesive bags, suprapubic aspiration (SPA), or catheterization. All these methods have considerable drawbacks. Adhesive bags may not adhere, may cause perineal discomfort, and are associated with high incidence of mixed cultures, which may reflect contamination. SPA is considered the best method to obtain urine for culture in children. It has been shown that the use of SPA guided by

Submitted, revised, 7 July 2003.

From the Department of Family Medicine, Sial Research Center for Family Medicine and Primary Care, Division of Community Health, Ben-Gurion University of the Negev, and Clalit Health Services, Southern District, Beer-Sheva, Israel. Address correspondence to Prof. Pesach Shvartzman, Chairman, Division of Community Health, Ben-Gurion University of the Negev, P.O. Box 653, Beer-Sheva, 84105, Israel (e-mail: spesah@bgumail.bgu.ac.il). portable ultrasound, in a children's hospital, increased the yield of successful taps from $52 \%$ to $79 \% .{ }^{3}$ However, this is a very intrusive and painful procedure and many physicians may feel uncomfortable using this method, especially in the primary care setting. Catheterization in children is considered painful and associated with discomfort, as in the geriatric population.

It is clear that there is a need for alternative methods for obtaining a urine sample. Recent studies assessed urine samples obtained from disposable diapers and compared the results with direct sampling. Ahmed et $\mathrm{al}^{4}$ demonstrated good agreement between urine cultures obtained from disposable diapers and urine cultures obtained from adhesive bags. Cohen et $\mathrm{al}^{5}$ showed high correlation between urine cultures obtained from disposable diapers and urine cultures obtained by SPA or catheter. These studies, however, did not evaluate ultra-absorbent gel-based diapers, which are in wide use in the United States, Europe, and Israel, and their sample size was very small (38 patients). Whitehall et $\mathrm{al}^{6}$ demonstrated a reliable bacterial culturing method from gel-based diapers soaked with suspensions of Escherichia coli.

In this study, we compared urine cultures from wetted ultra-absorbent gel-based diapers with urine samples obtained and examined by accepted meth- 
ods. Initially, the experiments conducted in our previous study ${ }^{6}$ were repeated with urine samples regularly sent to the microbiology laboratory of The Soroka Medical Center, Beer-Sheva, Israel. Thereafter, urine samples were compared with samples obtained from diapers soaked with the same urine, and examined 0 to 3 hours after soaking.

\section{Methods}

To determine the best method to extract a urine sample from gel-based diapers, the amount needed, and where in the diaper the sample should be taken from, the methods outlined in our previous study ${ }^{6}$ were repeated (diapers used: Depend Disposable Diapers; Shikma, Ltd., Ramle, Israel). Because a different diaper manufacturing company was used, the methods were reevaluated using positive urine cultures ( $\geq 10^{5}$ organism colony-forming units/ $\mathrm{mL}$ ) and negative urine samples routinely sent to the microbiology laboratory at Soroka Medical Center.

\section{Collection Method}

As described in our previous study, ${ }^{6}$ gel-based diapers were wetted with $10-\mathrm{mL}$ urine samples and then bisected in the inner side, along the long axis with sterile scissors; a sample of ultra-absorbent substance was taken by a 10 -inch ring gynecologic forceps; the sample was dropped into $9 \mathrm{~mL}$ of saline and vortexed for 10 seconds; a specimen of $0.01 \mathrm{~mL}$ was cultured on an agar plate (Figure 1). To assess different aspects of the technique, several experiments were performed. The lab technician culturing the urine from gel-based diapers was blinded to the urine samples results previously cultured by standard techniques.

\section{Results}

\section{Experiment 1}

Collecting methods from diapers were found to fully correlate with positive urine cultures (all 14 positive urine cultures were also positive from diapers). As found in our previous trial, ${ }^{6}$ no significant variations were found in the culturing results of the samples taken from different parts of the diaper (along the long axis: anterior, mid-line or posterior) (Figure 1).

\section{Experiment 2}

Full concordance of the culturing results was found between routine samples and the samples from the gel-based diapers, 0 to 3 hours after wetting (Table 1). In only one test was the result of the direct culture negative, and the gel-based diaper sample showed a mixed growth ( 3 hours after soaking). Thus, it was decided to culture urine from gelbased diapers up to 2 hours after wetting.

\section{Overall Comparison Experiments: Experiment 3}

Twenty-seven urine samples were cultured and compared with samples from diapers after wetting (Table 2). In 17 samples, there was no growth at all in both the diaper and the direct urine samples, and 6 cultures were positive. One culture showed a growth of 2 organisms, and in 3 samples, the growth was mixed/not clean, although not positive $\left(\geq 10^{5}\right)$, in both methods.

Taking into account the results of all the experiments performed (total of 60 experiments, Tables 1 to 2): 24 resulted in positive direct urine culture, 32 were negative, and 4 showed mixed growth. In the gel-based diaper cultures: 24 cultures were positive, 31 were negative, and 5 showed mixed growth. Thus, the sensitivity is $100 \%(24 / 24)$ and the specificity is $97 \%(31 / 32)$ (Table 3 ).

\section{Discussion}

The diagnosis of urinary tract infection is dependent on culturing a microorganism from urine. Ahmed et $\mathrm{al}^{4}$ showed that urine can be collected for culture from disposable diapers by extracting it from the wet fibers. They demonstrated good agreement in bacterial counts between urine collected this way and urine collected in adhesive bags. Their study also suggested that the contamination rate with this procedure might be lower than with the bag specimens. In another assessment of the reliability for microbiologic analysis of urine collection from disposable diapers of elderly women with severe urinary incontinence, it was found to be simple and reliable for routine analysis. No change over time in contamination or bacterial concentration was observed. ${ }^{7}$

Cohen et $\mathrm{al}^{5}$ compared cultures collected from disposable diapers with those collected by SPA or catheter. The results showed that all the infants in whom cultures collected by SPA or catheter were negative also had negative cultures from urine col- 


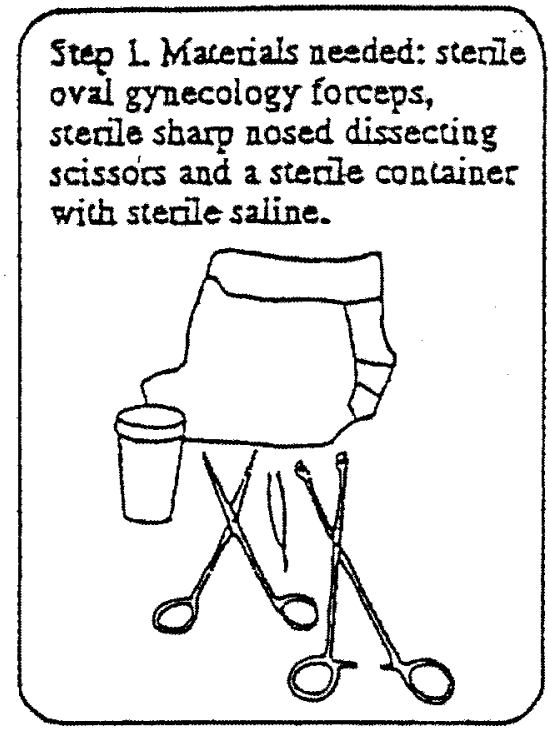

Step 2. Bisect the diaper along the long axis.

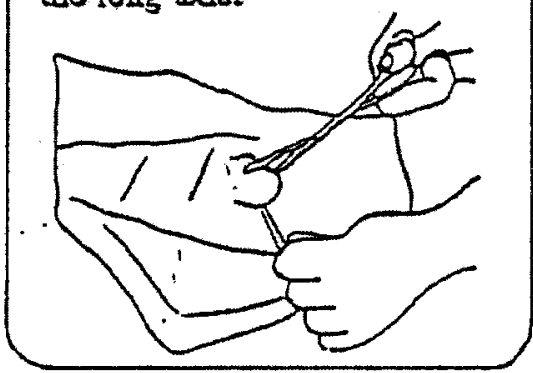

Step 3. Obtain a sample of the ultra-absocbent material with gynecology forceps.

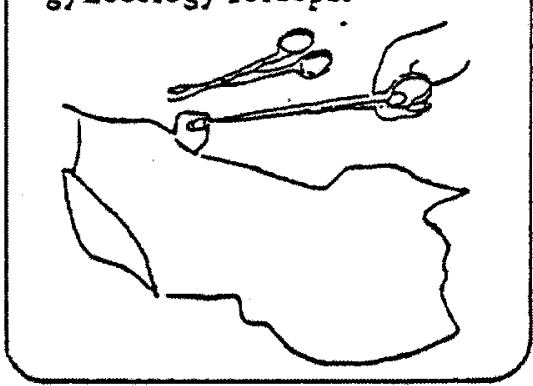

Figure 1. Extraction of a diaper sample for examination.

lected from diapers. Only 6\% (2 of 33) of the urine cultures collected from diapers were positive for E coli, with colony counts of $10^{5}$ colony-forming units/mL. Those 2 infants, however, had negative cultures from SPA and catheter specimens. Repeated urine cultures were negative, and the infants were not considered to have had a urinary tract infection. Thus, samples collected from disposable diapers were in complete agreement with those obtained by SPA or catheter in 36 of 38 cases
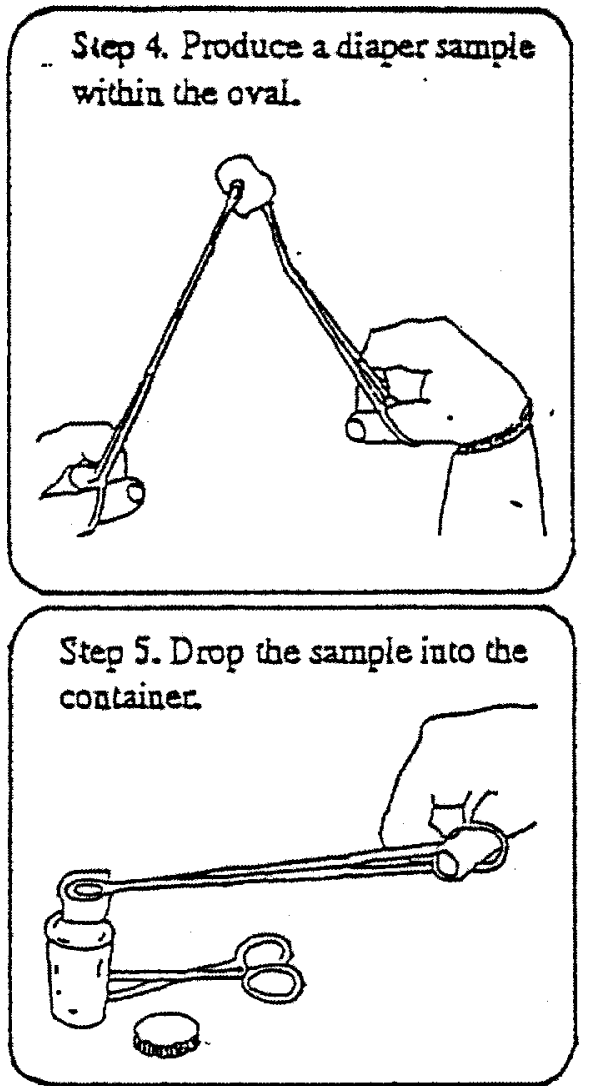

(95\%). A major limitation of their technique is that it is not applicable to ultra-absorbent gel-based diapers.

Recent research has shown that absorbent pads can be used yielding similar results to bag specimens. Twenty concurrent bag and pad specimens were collected from non-toilet-trained children. There was a lack of agreement between bag and pad specimens on both main outcome measures. Agreement between bag and pad specimens for the 
Table 1. The Effect of Delay before Culturing

\begin{tabular}{cll}
\hline Test & Urine Sample & Diaper Sample \\
\hline After 1 hour & & \\
1 & Negative & Negative \\
2 & Negative & Negative \\
3 & Mixed growth & Mixed growth \\
4 & Negative & Negative \\
5 & Positive & Positive \\
6 & Positive & Positive \\
After 2 hours & & \\
7 & Negative & Negative \\
8 & Negative & Negative \\
9 & Negative & Negative \\
10 & Negative & Negative \\
11 & Negative & Negative \\
After 3 hours & & \\
12 & Positive & Positive \\
13 & Negative & Negative \\
14 & Negative & Negative \\
15 & Negative & Negative \\
16 & Negative & Negative \\
17 & Negative & Mixed growth \\
18 & Negative & Negative \\
19 & Negative & Negative \\
\hline & & \\
& & \\
& & \\
& &
\end{tabular}

presence of white blood cells yielded a $\kappa=0.10$ (95\% CI: 0.19, 0.39), indicating poor agreement, whereas a $\kappa=0.5$ (95\% CI: $0.12,0.88)$ was calculated for the degree of agreement in bacterial growth reflecting moderate agreement. ${ }^{8}$ Another study evaluated the validity of using cotton gauze pad as a medium for collecting urine samples from young children and to examine the stability of the recoveries for creatinine and pesticide metabolites over 24 hours. The results suggest that cotton gauze pad is a promising candidate for collecting urine samples from young children wearing diapers for studies in which urinary pesticide metabolites are to be analyzed. ${ }^{?}$

In our study, experimentally culturing urine from ultra-absorbent gel-based diapers showed good concordance with direct sampling of urine collected from standard techniques. There were no false-positive or false-negative results, no influence of the gel substance on the culture result was found, and the procedure developed was simple, rapid, and practical for an outpatient setting.

The data from these experiments demonstrate that urine collected from gel-based diapers can closely predict the concentration of infected urine. Although the sample is small, the high correlation
Table 2. Comparison between Diaper Samples and Direct Cultures of Random Unknown Urine Samples

\begin{tabular}{|c|c|c|}
\hline \multirow[b]{2}{*}{ Test } & \multicolumn{2}{|c|}{ Overall } \\
\hline & Urine & Diaper \\
\hline 1 & Negative & Negative \\
\hline 2 & Negative & Negative \\
\hline 3 & Negative & Negative \\
\hline 4 & Negative & Negative \\
\hline 5 & Negative & Negative \\
\hline 6 & Negative & Negative \\
\hline 7 & Negative & Negative \\
\hline 8 & Negative & Negative \\
\hline 9 & Negative & Negative \\
\hline 10 & Negative & Negative \\
\hline 11 & Negative & Negative \\
\hline 12 & Two & Two \\
\hline 13 & Mixed & Mixed \\
\hline 14 & Mixed & Mixed \\
\hline 15 & Mixed & Mixed \\
\hline 16 & Positive & Positive \\
\hline 17 & Positive & Positive \\
\hline 18 & Negative & Negative \\
\hline 19 & Negative & Negative \\
\hline 20 & Negative & Negative \\
\hline 21 & Positive & Positive \\
\hline 22 & Positive & Positive \\
\hline 23 & Positive & Positive \\
\hline 24 & Negative & Negative \\
\hline 25 & Negative & Negative \\
\hline 26 & Negative & Negative \\
\hline 27 & Positive & Positive \\
\hline
\end{tabular}

coefficient $(\mathrm{R}=1.00)$ and the high sensitivity $(100 \%)$ and specificity (97\%), provide sufficient basis to proceed from the experimental laboratory phase to the clinical phase. Because another diaper brand was used (compared with our previous study ${ }^{6}$ ), generalization to other ultra-absorbent diapers is possible. The experimental protocol used still restricts immediate generalization to the clin-

Table 3. Summary of Experiments from Direct versus Random Unknown Urine Samples

\begin{tabular}{lrrcr}
\hline & \multicolumn{4}{c}{ Diaper } \\
\cline { 2 - 5 } Direct & Positive & Negative & Mixed Growth & Total \\
\hline Positive & 24 & 0 & 0 & 24 \\
Negative & 0 & 31 & 1 & 32 \\
Mixed growth & 0 & 0 & 4 & 4 \\
Total & 24 & 31 & 5 & 60 \\
\hline
\end{tabular}


ical setting for several reasons: first, the data show the ability of $E$ coli to survive exposure to the gel and to be readily extractable from the diaper. Although this is a potential limitation, it seems unlikely that other urine pathogens would behave differently. Second, the volume of urine voided by infants with UTI is unknown. However, it is important to remember that the bladder capacity of infants is surprisingly large. Term newborns have 50 to $60 \mathrm{~mL}$ of bladder capacity, and this increases to $100 \mathrm{~mL}$ by the end of the first year. It seems likely that there is frequent bladder emptying in infants with UTI. Because weight measurements suggest that even 10 to $20 \mathrm{~mL}$ will yield reproducible results with our technique, this should not represent a clinical limitation.

Other potentially relevant issues are not addressed by these experiments. Diapers worn by infants are in direct contact with perineum and could be contaminated. Clearly, gross fecal soiling would interfere with our technique. Previous studies with disposable diapers surprisingly indicate that contamination concerns might not be a major problem. ${ }^{4-7}$ There are a host of other issues to be considered. What impact does the gel have on microbiologic selection? Does it affect some organisms (either as a growth media or as an inhibitor or toxin to the bacteria) but not others? What influence does urine $\mathrm{pH}$ have? Does it interact with blood (eg, in hematuria with UTI)? The study does not address these. Because this is only a preliminary study, studies investigating this novel technique of diaper culture in the clinical setting (both in the infant and geriatric populations) should be conducted. Given the few patients testing positive, a larger sample would be needed. Furthermore, set criteria for standardizing the collection method should be applied: no stool in diaper and last diaper change was up to 2 hours from collection (even though in the laboratory 3 hours showed good results).

\section{References}

1. Kunin CM. Detection, prevention and management of urinary tract infections. Philadelphia: Lea and Febiger; 1987.

2. Ouslander JG, Schapira M, Schanelle JF. Urine specimen collection from incontinent female nursing home residents. J Am Geriatr Soc 1995;43:279-81.

3. Gochman RF, Karasic RB, Heller MB. Use of portable ultrasound to assist urine collection by suprapubic aspiration. Ann Emerg Med 1991;20:631-5.

4. Ahmad T, Vickers D, Campbell S, Coulthard MG, Pedler S. Urine collection from disposable nappies. Lancet 1991;338:674-76.

5. Cohen HA, Woloch B, Linder N, Vardi A, Barzilai A. Urine samples from disposable diapers: an accurate method for urine cultures. J Fam Pract. 1997; 44:290-2.

6. Whitehall J, Shvartzman P, Miller MA. A novel method for isolating and quantifying urine pathogens collected from gel-based diapers. J Fam Pract 1995;40:476-9.

7. Belmin J, Hervias Y, Avellano E, Oudart O, Durand I. Reliability of sampling urine from disposable diapers in elderly incontinent women. J Am Geriatr Soc. $1993 ; 41: 1182-6$.

8. Farrell M, Devine K, Lancaster G, Judd B. A method comparison study to assess the reliability of urine collection pads as a means of obtaining urine specimens from non-toilet-trained children for microbiological examination. J Adv Nurs 2002;37:387-93.

9. Hu YA, Barr DB, Akland G, et al. Collecting urine samples from young children using cotton gauze for pesticide studies. J Expo Anal Environ Epidemiol 2000;10:703-9. 